\title{
Value of the 6 minute walk test in predicting multi vessel coronary arterial disease
}

\author{
Ruvan Ekanayaka, Yasindu Waniganayake \\ Institute of Cardiology, National Hospital of Sri Lanka, Colombo, Sri Lanka. \\ Email: ruvan_nishali.ekanayaka@yahoo.com
}

Received 12 April 2013; revised 12 May 2013; accepted 20 May 2013

Copyright (C) 2013 Ruvan Ekanayaka, Yasindu Waniganayake. This is an open access article distributed under the Creative Commons Attribution License, which permits unrestricted use, distribution, and reproduction in any medium, provided the original work is properly cited.

\begin{abstract}
The 6 minute walk test (6MWT) is well established in the clinical assessment of heart failure, pulmonary hypertension and COPD. Its value as a submaximal stress test in the risk stratification of chronic stable ischaemic syndromes is as yet not validated. 95 patients undergoing coronary angiography for assessment of chronic stable angina performed the 6MWT according to a modified protocol. The gamma correlation test indicated a moderately significant relationship between ECG changes plus symptoms at the end of the 6MWT and multi vessel coronary arterial disease. The $T$ wave changes showed no significant correlation. Hence the 6MWT is a useful tool in the risk stratification of stable ischaemic syndromes which can be safely performed in a general ward prior to hospital discharge. It would be a useful preliminary test before planning a programme of cardiac rehabilitation.
\end{abstract}

Keywords: Coronary Angiogram; 6 Minute Walk Test; Multi Vessel Coronary Disease

\section{INTRODUCTION}

Exercise testing is a well-established mode of noninvasive investigation used frequently in the setting of cardiac and pulmonary disease as a test of functional status. It has value in two aspects of cardiac rehabilitation. The first is that it provides reproducible data which helps the clinician risk stratify patients more precisely. This becomes important when costly procedures which have limited availability need to be reserved for patients who would profit most from them in terms of prognostic benefit. The second is that exercise testing contributes towards the physical and mental rehabilitation of cardiac patients, as supervised exercise helps build up patient confidence in engaging in an exercise programme on a domiciliary basis [1].

In cardiac practice, formal stress testing employing the treadmill or bicycle ergometer is standard procedure for stable ischaemic syndromes. The specificity and sensitivity of routinely used stress test protocols are well established, but the values differ widely depending on the ECG criteria used and patient population studied [2]. However formal stress testing necessitates the availability of a specially set up exercise laboratory with sophisticated equipment with trained technical and medical staff in attendance. This involves considerable cost and would generate long waiting lists if such resources are limited. The inability to subject patients to stress testing within the time schedule recommended in management protocols $[3,4]$, often delays proper risk stratification. Hence, high risk patients may not be identified sufficiently early in order to subject them to coronary intervention or surgery.

It has been customary in many departments to assess the ischaemic patients' predischarge exercise capacity using nonformal exercise tests such as climbing two flights of stairs or doing two brisk walks around the ward. Such nonstandard exercise tests are commonly utilized by physicians merely to overcome the difficulty in obtaining a formal stress test in the predischarge period.

One commonly used test of functional capacity is the 6 minute walk test (6MWT). This is frequently performed without following a specific protocol. Although the 6MWT has been used by clinicians for patients who are unable to undergo the formal stress tests due to orthopaedic or rheumatologic pathology it appears that this was primarily to obtain an estimation of the patient's functional capacity rather than assess the ischaemic status [5].

So far as cardiac diseases are concerned, a large body of literature exists where the 6MWT has been studied with respect to heart failure and pulmonary hypertension [6-8]. However there seems to be a paucity of published 
studies where the 6MWT has been used as a substitute for formal exercise testing in patients with established coronary ischaemia.

In a recently published cohort analysis, the Heart and soul study investigators reported that the 6MWT was as good as the formal stress test in predicting death, myocardial infarction and heart failure in patients with stable coronary disease, which conclusion seems to justify the use of the 6MWT in risk stratification of patients with stable coronary arterial disease [9].

The present study was conducted in order to investigate a specific protocol of the 6MWT in correlation with coronary angiographic data in order to validate the $6 \mathrm{M}$ WT as a non invasive tool in risk stratifying patients with multivessel coronary artery disease.

\section{MATERIALS AND METHODS}

Hundred consecutive patients admitted for coronary angiography were included in the study. All patients had stable coronary arterial disease for which treadmill exercise testing following the Bruce protocol had been performed subsequent to which the treating cardiologist had decided on coronary angiography. A 6MWT was performed as a pre discharge test on the day following coronary angiography.

The following exclusion criteria were applied.

1. Rest angina and/or acute coronary syndrome within the past 6 weeks.

2. Significant arrhythmia:

1) Heart rate $<50 \mathrm{bpm}$ or $\geq 100 \mathrm{bpm}$ at rest;

2) Non sustained or sustained VT;

3) Atrial fibrillation or flutter;

4) $2^{\circ}$ or $3^{\circ}$ heart blocks;

5) Multifocal or R on T VE;

6) Unifocal VE or AE, occurring more than 10/minute;

3. LV ejection fraction of $<35 \%$.

4. Structural heart disease.

5. Dyspnoea of pulmonary aetiology which precludes brisk walking.

6. Orthopaedic or rheumatologic pathology which precludes brisk walking.

7. History of hospitalization for an acute cardiac syndrome during the past 3 months.

All patients were on constant medication for at least one month preceding the $6 \mathrm{MWT}$.

\subsection{MWT Protocol}

- The patients fitness to walk was assessed by questioning them regarding angina at rest, severe muscle/joint pain.

- The test objective was explained to the patient as follows: "This test will try to determine your ability to do moderate exercise so that you will be confident to perform most of your day to day activities at home. In addition we hope to detect whether your ECG shows up any changes while you engage in this test. If you develop any chest pain, difficulty in breathing or any other new symptom, stop walking and the staff will come to you. You may sit down on the closest bed or chair whenever you wish."

- The patient was shown the ward corridor (which was 35 feet in length) in which he would be required to walk for 24 lengths (to cover a total distance of 840 feet). This works out to a walk speed of $2.5 \mathrm{~km} /$ hour which is the speed used in the second stage of the Bruce Protocol but without the inclination used in the treadmill test. He was then instructed to commence walking and verbal encouragement was given such as "You are doing well. Walk a little faster if you can." whenever the specifically allocated supervising medical officer deemed the speed as being too slow.

- The heart rate and BP recordings were made by a single medical officer and the 12 lead ECG was recorded before and soon after the completion of the 6MWT.

- Patients were allowed to walk barefoot or use suitable footwear in accordance with their preference.

- A light meal 1 hour before the test was acceptable.

- The routine drugs were administered uninterrupted. (i.e. the test was performed on fully medicated patients.)

- If the patient complained of chest pain, an attempt was made to assess whether it was true angina or nonspecific chest pain. If nonspecific chest pain was more likely, the patient was encouraged to proceed.

- If the patient complained of difficulty in breathing, it was taken to be significant if 3 or more on the Borg Scale.

The literature reports that the 6MWT is sometimes conducted in a "self-controlled" format where the Borg scale is used to ensure that all the subjects exercise up to sub maximal level [10]. Although this would be appropriate for heart failure patients where the functional capacity is being assessed it leads to differing distances being covered during the 6 minutes period, which makes comparison with the formal treadmill test results difficult. Hence the self-controlled format was not followed in the present study.

\subsection{Statistical Analysis}

Continuous variables such as pulse rate, blood pressure were reported as a percentage increase or decrease between pretest and posttest values. Symptoms and new ECG changes that occurred at the end of the 6 minutes 
walk test were reported as string variables. The data of Exercise ECG and coronary angiography of each subject were also reported as string variables.

$\mathrm{X}^{2}$ analysis was used to assess statistically significant relationships between 6MWT and results of coronary angiography (CA) and Exercise ECG. Statistical differences with a $P$ value $<0.05$ were considered significant.

Gamma correlation test was used to detect correlations between the results 6MWT, CA and Exercise ECG results in the total sample.

One way Analysis of variance (ANOVA) was used to determine whether there was any significant differences in means of continuous variables (pulse rate and BP) in each CA result group.

The string variables were converted in to ordinal variables with a hierarchy of values according to severity. Exercise ECG/coronary angiogram results of each subject were also reported as string variables and as ordinal variables with a hierarchy according to severity. The hierarchy given for each set of data is as given in Table 1.

Spearman's rank correlation coefficient was also used to assess correlations between the 6MWT results and CA/Exercise ECG results.

Taking the CA result as the gold standard test to detect multivessel CAD, the patients were divided in to multi vessel disease (DVD/TVD) and non multi vessel disease (normal CA/minor disease/SVD) categories (Table 2). The sensitivity, specificity, positive predictive value and negative predictive value were calculated for each of the 6MWT variables and for combinations of 6MWT variables (ECG changes + symptoms, ST segment changes + symptoms) as given in Table 7.

\section{RESULTS}

Out of the initial 100 patients, 5 were not included due to incomplete data. Hence the data of 95 patients were analyzed using SPSS (statistical package for social sciences) version 15.

\subsection{Baseline Characteristics}

Age of subjects ranged from $33-75$ years (Mean 55 years). $18(18.9 \%)$ were females and $77(81.1 \%)$ were males (Table 3).

At the end of the 6 minutes walk test, the mean pulse rate rise was $20.3 \%$, mean systolic blood pressure rise was $2.4 \%$ and the mean diastolic blood pressure rise was $1.2 \%$.

$50.5 \%$ of the subjects developed new ECG changes which were interpretable as indicating cardiac ischaemia.

At the end of 6 minutes walk test $50.5 \%$ developed test induced symptoms. The symptom breakdown is given in Table 4.

\subsection{Interpretation of the 6MWT ECG}

The following were taken to constitute a positive effect.

1. De novo $\mathrm{T}$ wave inversion of $\geq 0.5 \mathrm{~mm}$ in two or more leads attributable to the distribution of a single coronary artery.

2. ST segment depression of $\geq 0.5 \mathrm{~mm}$ in anterior leads and $\geq 1 \mathrm{~mm}$ in inferior leads (Measured $80 \mathrm{msec}$ past the J point).

3. ST segment elevation of $\geq 1 \mathrm{~mm}$ above that at baseline in leads showing a preexisting pathological Q wave.

Amplitude of the $\mathrm{R}$ wave, change in the $\mathrm{P}$ wave configuration, inversion of $U$ waves, QTc interval changes, arrhythmias were studied but were not detected in any patient. All ECGs were read by a single consultant cardiologist.

\subsection{Interpretation of the Coronary Angiogram}

The number of coronary vessels diseased was defined by the index vessels: the left anterior descending (LAD), the circumflex, and the right coronary arteries (or distal circumflex in left dominance). $70 \%$ reductions in luminal diameter in the proximal or middle segment of all three arteries were taken to indicate three vessel disease. The middle segments included major branches arising from middle segments of an index vessel, e.g. diagonal, an obtuse marginal, or a posterior descending artery (or distal segment in the case of the LAD). All coronary angiograms were interpreted by two cardiologists. Depending on the number of index vessels diseased the patients were determined to have a single (SVD), double (DVD) or triple (TVD) vessel disease.

The demographic data and 6MWT results of patients in each CA result category are given in Table $\mathbf{5}$.

\section{4. $X^{2}$ Analysis}

A significant association was found between the coronary angiography results and Exercise ECG results $(P<$ $0.001)$.

There were no significant associations between CA results and the clinical parameters measured in the 6MWT, namely pulse rate difference, BP change and symptoms.

\subsection{Study of Correlations Between 6MWT Results/ExECG and CA Results}

A moderately and significant correlation was found between the composite of all ECG changes plus symptoms with CA results $(P<0.01)$.

1. The ST segment changes of 6MWT with CA results $(P<0.05)$.

2. The Ex ECG results with CA results $(P<0.01)$. 
Table 1. String/continuous numbers allocated for variables in the 6MWT, Ex ECG and coronary angiogram.

\begin{tabular}{|c|c|c|c|c|c|}
\hline ECG changes after $6 \mathrm{MWT}$ & String variable & $\begin{array}{l}\text { Continuous } \\
\text { variable }\end{array}$ & Symptoms after $6 \mathrm{MWT}$ & $\begin{array}{c}\text { String } \\
\text { variable }\end{array}$ & $\begin{array}{l}\text { Continuous } \\
\text { variable }\end{array}$ \\
\hline Pre and post ECG both normal & 0 & 0 & No symptoms & 0 & 0 \\
\hline No new changes in post ECG & 1 & 1 & Mild chest pain & 1 & 1 \\
\hline Resolution in post ECG & 2 & 2 & Shortness of breath & 2 & 2 \\
\hline New changes in post ECG & 3 & 3 & Angina & 3 & 3 \\
\hline Summary of 6MWT ECG changes & & & Dizziness & 4 & 4 \\
\hline No new changes/both normal & 0 & 0 & Summary of symptoms & & \\
\hline New changes/resolution & 1 & 1 & Symptoms & 0 & 0 \\
\hline T wave changes in 6MWT ECG & & & No symptoms & 1 & 1 \\
\hline Normal in both pre/post ECG & 1 & 1 & $\begin{array}{l}\text { Summary of symptoms and } \\
\text { ECG changes of } 6 \mathrm{MWT}\end{array}$ & & \\
\hline No new change & 2 & 2 & Symptoms $^{\circ}$ ECG change $e^{+}$ & 0 & 0 \\
\hline $\mathrm{T}$ wave inversion $<0.5 \mathrm{~mm}$ & 3 & 3 & Symptoms $^{+}$ECG change ${ }^{\circ}$ & 1 & 1 \\
\hline T wave $0.5-1 \mathrm{~mm}$ & 4 & 4 & Symptoms $^{0}$ ECG change ${ }^{+}$ & 2 & 2 \\
\hline $\mathrm{T}$ inversion $>1 \mathrm{~mm}$ & 5 & 5 & Symptoms $^{+}$ECG change ${ }^{+}$ & 3 & 3 \\
\hline ST segment Changes in 6MWT ECG & & & Coronary Angiogram results & & \\
\hline Normal in both pre/post ECG & 1 & 1 & No occlusion & $\mathrm{n}$ & 1 \\
\hline Deepening of ST depression & 2 & 2 & Minor disease & $\mathrm{m}$ & 2 \\
\hline $\begin{array}{l}\text { Normalizing of ST depression/elevation } \\
\text { (Difference }>1 \mathrm{~mm} \text { ) }\end{array}$ & 3 & 3 & SVD & s & 3 \\
\hline New ST depression in post ECG & 4 & 4 & DVD & $\mathrm{d}$ & 4 \\
\hline New ST elevation in post ECG & 5 & 5 & TVD & $\mathrm{t}$ & 5 \\
\hline Exercise ECG results & & & $\begin{array}{l}\text { Pulse rate change/systolic BP } \\
\text { change/diastolic BP change } \\
\text { after } 6 \mathrm{MWT}\end{array}$ & String variable & \\
\hline Inconclusive & 0 & 0 & No change & 1 & \\
\hline Stage 1 & 1 & 4 & Rise & 2 & \\
\hline Stage 2 & 2 & 3 & Fall & 3 & \\
\hline Stage 3 & 3 & 2 & & & \\
\hline Stage 4 & 4 & 1 & & & \\
\hline Positive on recovery & 5 & 1 & & & \\
\hline
\end{tabular}

Table 2. Comparison of coronary angiographic findings and 6MWT results.

\begin{tabular}{lccc}
\hline \multicolumn{1}{c}{ 6MWT result } & $\begin{array}{c}\text { Multivessel disease category } \\
\text { (DVD, TVD) }\end{array}$ & $\begin{array}{c}\text { Non multivessel disease category } \\
\text { (normal, minor disease, SVD) }\end{array}$ & Total \\
\hline ECG positive & 37 & 11 & 48 \\
ECG negative & 30 & 17 & 47 \\
ST segment positive & 21 & 4 & 25 \\
ST segment negative & 46 & 24 & 70 \\
T wave positive & 29 & 12 & 41 \\
T wave negative & 38 & 16 & 54 \\
Symptoms positive & 35 & 13 & 48 \\
Symptoms negative & 32 & 15 & 47 \\
Symptoms and ECG positive & 52 & 18 & 70 \\
Symptoms and ECG negative & 15 & 10 & 25 \\
Symptoms and ST segment positive & 45 & 16 & 61 \\
Symptoms and ST segment negative & 22 & 12 & 34 \\
Total & $\mathbf{6 7}$ & $\mathbf{2 8}$ & $\mathbf{9 5}$ \\
\hline
\end{tabular}


Table 3. Characteristics of the study population.

\begin{tabular}{lc}
\hline \multicolumn{1}{c}{ Male/female } & $77 / 18$ \\
\hline Age & $57 \pm 4$ years \\
HT & $22 \%$ \\
Valvular lesions & $0 \%$ \\
Cardiomyopathies & $0 \%$ \\
EF $\%$ & $55 \pm 5$ \\
Medical therapies & \\
Beta blockers & $81 \%$ \\
Rate limiting CCB & $19 \%$ \\
Nitrates & $97 \%$ \\
ACE inhibitors & $86 \%$ \\
Antiplatelet agents & $100 \%$ \\
\hline
\end{tabular}

Table 4. Breakdown of symptoms experienced during the $6 \mathrm{M}-$ WT.

\begin{tabular}{lc}
\hline \multicolumn{1}{c}{ Symptom } & Frequency (\%) \\
\hline No symptoms & $47(49.5 \%)$ \\
Shortness of breath & $12(12.6 \%)$ \\
Mild chest pain & $10(10.5 \%)$ \\
Angina & $22(23.2 \%)$ \\
Dizzinness & $4(4.2 \%)$ \\
\hline
\end{tabular}

3. There was no significant correlation of $\mathrm{T}$ wave changes of the 6MWT with CA results.

After conversion of string variables into continuous variables with values set according to a hierarchy of ascending severity, Spearman's rank Correlation Coefficient was applied. The associations found were similar to those of the gamma correlation test Table 6.

\subsection{The Sensitivity, Specificity, Positive and Negative Predictive Values}

The ST segment changes of the 6MWT gave the highest specificity $(86 \%)$ and positive predictive value $(84 \%)$ of diagnosing multi vessel CAD (Table 7). However, the sensitivity (31\%) and negative predictive value (34\%) of both were low. The composite of all ECG changes (i.e. ST and T changes) had the next highest positive predictive value $(77 \%)$, negative predictive value $(36 \%)$ and specificity $(61 \%)$ even though the sensitivity was low $(55 \%)$.

Of the 6MWT variables, the composite of all ECG changes plus symptoms showed the highest sensitivity $(78 \%)$ and the highest negative predictive value (40\%). The positive predictive value was also moderately high (74\%), even though the specificity was low (36\%).

The $\mathrm{T}$ wave changes had low sensitivity $(43 \%)$ specificity $(57 \%)$ and negative predictive value $(29.62 \%)$.

Overall, when considering all the 6MWT variables, the positive predictive values were moderately high, with the maximum being $84 \%$ and minimum being $71 \%$. The negative predictive values were generally low, with the maximum being $40 \%$ and minimum being $30 \%$. The sensitivity varied from $31 \%$ to $78 \%$ and specificity varied from $36 \%$ to $86 \%$.

\section{DISCUSSION}

Risk stratification of patients with acute ischaemic heart disease is a continuous process. However there are three instances deserving special attention, namely, 1) the time of presentation, 2) the period of hospitalization, and 3) the time of hospital discharge [5].

Stress testing has no place in the first two instances but

Table 5. Distribution of 6MWT variables in angiographically determined coronary artery disease categories.

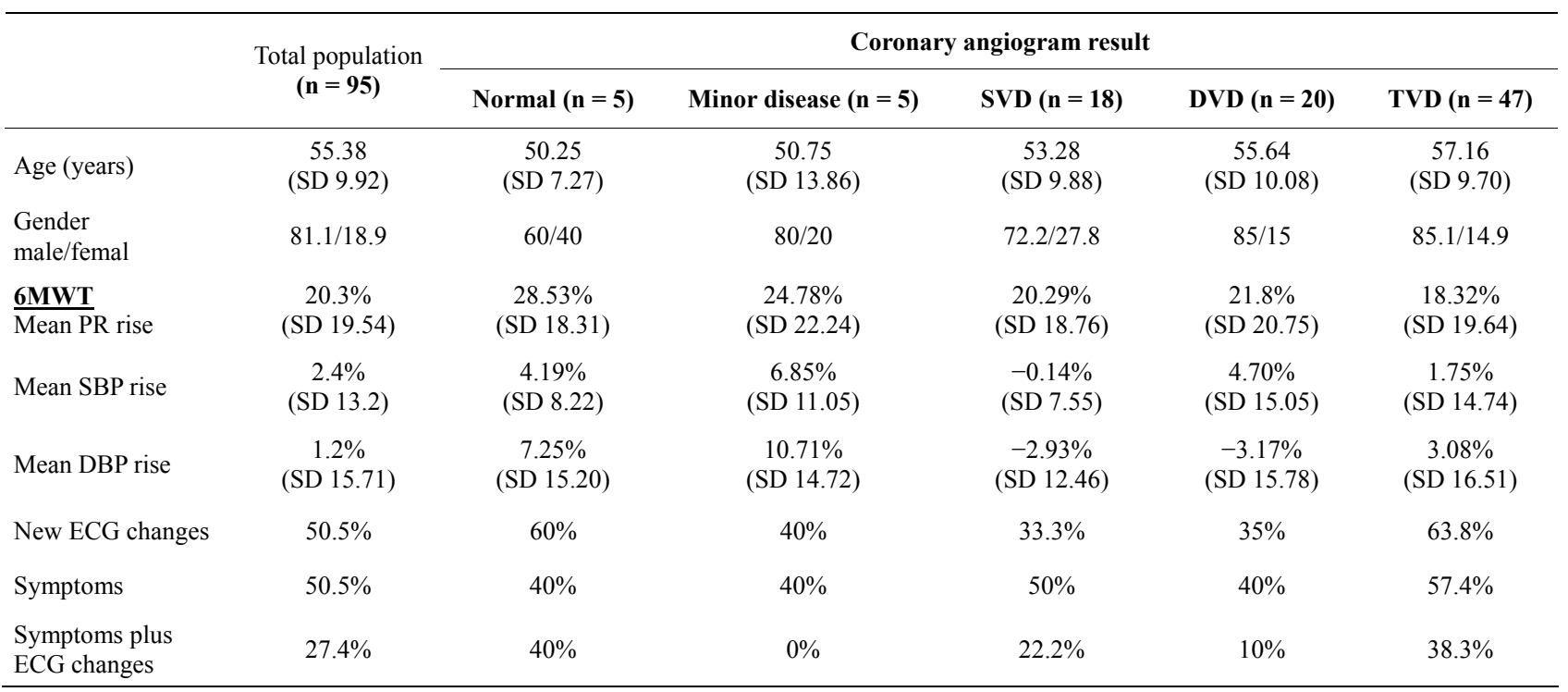


Table 6. Correlations of 6MWT/Ex ECG variables with presence of multivessel coronary arterial disease.

\begin{tabular}{lcc}
\hline & Gamma & Spearman \\
\hline ExECG-ECG changes. & $0.513^{* *}$ & $0.401^{* *}$ \\
ECG changes of 6MWT (ST + T & $0.341^{*}$ & $0.221^{*}$ \\
wave changes) & & \\
Symptoms of 6MWT & 0.249 & 0.190 \\
ECG changes + symptoms of 6MWT & $0.290^{*}$ & $0.244^{*}$ \\
ST segment changes of 6MWT & $0.360^{*}$ & $0.226^{*}$ \\
\hline${ }^{*} P<0.05 ;{ }^{* *} P<0.01$ & &
\end{tabular}

Table 7. Sensitivity, specificity predictive values of the 6MWT in relation to multi vessel coronary arterial disease.

\begin{tabular}{lcccc}
\hline \multicolumn{1}{c}{ 6MWT result } & Sensitivity & Specificity & $\begin{array}{c}\text { Positive } \\
\text { predictive } \\
\text { value (\%) }\end{array}$ & $\begin{array}{c}\text { Negative } \\
\text { predictive } \\
\text { value (\%) }\end{array}$ \\
\hline ECG & 0.55 & 0.61 & 77.03 & 36.17 \\
$\begin{array}{l}\text { ST segment } \\
\text { changes }\end{array}$ & 0.31 & 0.86 & 84.00 & 34.0 \\
T wave changes & 0.43 & 0.57 & 70.73 & 29.62 \\
$\begin{array}{l}\text { Symptoms } \\
\text { Symptoms + ECG }\end{array}$ & 0.52 & 0.54 & 72.90 & 31.91 \\
$\begin{array}{l}\text { Symptoms + ST } \\
\text { segment changes }\end{array}$ & 0.67 & 0.36 & 74.28 & 40.00 \\
\hline
\end{tabular}

has been included in many predischarge assessment protocols .It is generally recognized that three factors have major influence on the prognosis of patients discharged from hospital i.e. 1) the resting LV function which probably indicates the quantum of muscle permanently damaged, 2) viable myocardium which is at risk of future acute events and depends on the extent and severity of coronary lesions, and 3) susceptibility to develop serious ventricular arrhythmias. It is with relation to the second prognostic determinant that stress testing provides valuable data for the clinician.

The present study is concerned with the clinical value of the 6MWT in detecting patients with multi vessel coronary artery disease. No noninvasive test is successful in predicting the severity of atherosclerotic disease with $100 \%$ sensitivity. Even the commonly performed treadmill test which is used for diagnostic as well as prognostic evaluation has a sensitivity and specificity of $68 \%$ and $70 \%$ respectively [11]. The Dukes treadmill score, derived from ST changes, symptoms of angina and exercise duration assists clinicians to objectively assess the stress test and better predict the presence of occlusive coronary arterial disease. When the Dukes treadmill score (DTS) was high $(\leq-11), 74 \%$ had $3 \mathrm{VD}$ or significant LM disease. Thus even the formal treadmill test when analyzed using the DTS gives only a moderate correlation with the coronary angiographic findings. In fact when the alternative Lauer score is used, which is believed by some authorities to be more accurate than the Dukes score, approximately $21 \%$ of participants had to be reclassified in to different risk categories $[12,13]$.

In the present study, the 6MWT was analyzed using two of the DTS variables (i.e. ST changes and symptoms) as the third variable of exercise duration was not relevant in this study as the duration was fixed in the 6MWT protocol.

Bayes' theorem states that the probability of a positive result is affected by the conditional probability of a positive test result in the study population. Hence the results of the present study must be considered as valid in patients with high probability of coronary arterial disease.

\subsection{Correlation of Symptoms and CA Findings}

Post-6MWT symptoms showed no significant association with the CA results. The 6MWT in our study was performed while the patients were on full anti anginal medication, which would have mitigated the symptoms to a significant degree leading to the non-significant correlation of symptoms with the coronary angiographic findings.

\subsection{Correlation of ECG Changes and CA Findings}

ECG changes of the 6MWT showed significant correlation with CA results, when the $\mathrm{T}$ wave changes plus ST segment changes are considered together. However when the $\mathrm{T}$ wave changes and ST segment changes are considered separately, ST segment changes alone were significantly correlated to the CA results. The $\mathrm{T}$ wave changes show no correlation at all and hence in identifying multi vessel disease, only the ST changes would be useful. ST segment changes can be caused by many non ischaemic pathologies which would have been excluded in the initial workup of the study patients.

The composite of symptoms plus all ECG changes gives a very significant correlation with multi vessel coronary artery disease.

Medication could have improved the ischaemic response of the myocardium in our study population and because the present study was done on patients on full medication, we have used a ST depression of $0.5 \mathrm{~mm}$ as the cut off, as some other workers have used this value for patients on beta blocker therapy [14].

\subsection{The Sensitivity, Specificity, Positive and Negative Predictive Values}

When the 6MWT is used for risk stratification before discharge, it is best to use the composite of all ECG changes plus symptoms, to predict the presence of multi vessel CAD (sensitivity 78\%, Positive predictive value of $74 \%$ ). Among all the 6MWT variables, all ECG chan- 
ges plus symptoms also had the highest negative predictive value of $40 \%$, which is actually a low value and therefore the importance of screening all the patients for multi vessel CAD depending on the availability of facilities, should be emphasized. A specificity of $86 \%$ and a positive predictive value of $84 \%$ seen when the ST segment changes are employed to diagnose multi vessel $\mathrm{CAD}$, will clinically relevant for identifying the patients requiring immediate coronary intervention following a myocardial infarction.

\subsection{Safety}

The present study clearly demonstrates that the 6MWT test is safe to be performed in a general ward. Although other investigators suggest using a 100 feet hallway [15] we used the usual 35 feet corridor of a general cardiac ward for ease of observation, as in this scenario the patient is always within the view of the nursing officer, electro cardiac technician or medical officer. Thus visual supervision is ensured as patients are not electrically monitored during the performance of the 6MWT.

It is important also that patients be able to immediately communicate with the responsible staff if they were to experience any discomfort. For these reasons our study was conducted using a 35 foot corridor, the only drawback being that the patient has to make frequent turns breaking a smooth continuous walk.

\subsection{Limitations and Improvements}

The obvious limitation of the 6MWT is that it is limited in time utilizing 4 - 6 mets on average whereas the Bruce protocol stress test extends up to 12 minutes utilizing 16 - 18 mets. The walk test has been extended to 12 minutes by some workers which may be a better test for detecting moderate risk patients. However the time taken for each patient is greater, and hence the number of patients that could be accommodated within a given period of time would be correspondingly fewer. Five versions of the walk test have published namely $2,3,5,6,12$ minute walk tests [16], but the 6MWT appears to be a practical choice. Studies have confirmed its value in PPHT, HF and several pulmonary conditions. The present study shows the 6MWT to be of value as a sub maximal stress test for predicting multi vessel coronary disease.

If facilities permit, telemetric monitoring of the patient for the duration of the 6MWT could be performed specifically when patients with arrhythmias are studied. This is performed in our intermediate coronary care unit but is not routine practice. However it would be an improvement in the 6MWT test.

The 6MWT does not assess the patient's functional capacity or ischaemic burden at maximal exercise but at sub maximal levels of exertion [15]. This may not nec- essarily detract from its value in risk stratification, for it could be reasonably argued that high risk patients would manifest changes at low quanta of exercise compared to patients with low burden of atherosclerotic disease. Clinical experience however indicates that this not always so.

The 6MWT, as performed in accordance with the recommendation of the American thoracic society (2002) allows the patient to use his own speed, the instruction being "walk as fast as possible for 6 minutes" without the velocity being specified [15]. The patient determines his own speed. This would affect the sensitivity of the test if the patient opts for a slow speed due to psychological factors (fear of walking too fast etc.). Furthermore, the test results may not be reproducible or comparable as different patients will use diverse velocities. This would not be the case when performing the formal stress test using the Bruce protocol, where the speeds are fixed and individual variation is not allowed. Hence when developing the protocol for the present study it was thought that the $6 \mathrm{MWT}$ would be more robust if the speed was predetermined.

The speed selected for the 6MWT in our study was 2.5 $\mathrm{km} / \mathrm{hr}$. This corresponds to the speed used in the 2 nd stage of the Bruce protocol but without the $12 \%$ grade inclination. The energy expenditure involved would be approximately $4-6$ mets which is the energy level used in day to day activities. This fact eliminates the need for familiarization with the 6MWT test as has been done in some studies. It has been demonstrated that familiarization with the test does not affect the reproducibility of results, namely heart rate, systolic BP, and ventilatory responses [17], although some other investigations have reported a learning effect [18].

\subsection{Prognostic Significance}

The 6MWT has been shown to have a strong prognostic significance in primary pulmonary hypertension [19]. Guazzi et al. demonstrated a correlation between the 6 MWT and the variables of CEPT (i.e. Peak $\mathrm{VO}_{2}, \mathrm{VO}_{2}$ at anaerobic threshold and $\mathrm{VE} / \mathrm{VO}_{2}$ slope, $\mathrm{EoB}$ ) in a cohort of patients with heart failure (N-SHA class $2.2 \pm 0.75$ ), but were unable to demonstrate that the 6MWT correlated with the 4 year mortality compared to CEPT variables which did [20]. Hence the prognostic power of the 6MWT differs for each clinical entity.

Data from our study indicates that a category of ischaemic patients with multi vessel coronary arterial disease can be identified by the 6MWT but no prognostic inferences can be made from this data alone. The recent study from the Heart and soul study group however provides data indicating the prognostic value of the 6MWT. 


\section{CONCLUSIONS}

The 6 MWT has sensitivity comparable with the standard stress test when the composite of ECG changes plus symptoms is used for analysis. Although its results give satisfactory correlation with the coronary angiographic determination of multi vessel coronary arterial disease the sensitivity is too low in order for it to be used as the sole criterion in deciding in favour of invasive investigation.

The 6MWT would be a simple tool to evaluate the cardiac patient prior to discharge from the hospital enabling the attending physician to reliably inform the patient of the quantum of physical exertion he could safely undertake in the form of day to day activities or exercise. The 6MWT as performed in this study would be a useful tool for preliminary risk stratification while awaiting more formal stress testing using the full Bruce protocol and in planning and individualizing a cardiac rehabilitation programme for each patient.

\section{REFERENCES}

[1] Kappagoda, C. (1984) Rehabilitation after myocardial infarction. Medical Examination Publishing Co., Inc., New York, 72.

[2] Goldschlager. N., Selzer, A. and Cohn, K. (1976) Treadmill stress tests as indicators of presence and severity of coronary artery disease. Annals of Internal Medicine, 85, 277. doi:10.7326/0003-4819-85-3-277

[3] ESC (2007) Compendium of ESC guidelines 2007. Lippincott Williams and Wilkins, 80.

[4] Fuster, V., Ed. (2009) The AHA guidelines and scientific statements handbook. Wiley-Blackwell, Dallas, 81.

[5] Robert, O.B., Douglas, L.M., Douglas, P.Z., Peter, L. and Braunwald, E., Eds. (2012) Braunwald's heart disease. 9th Edition, Elsevier Saunders, Philadelphia, 171, 1159.

[6] Olsson, L.G., Swedberg, K., Clark, A.L., Witte, K.K. and Cleland, J.G. (2005) Six minute corridor walk test as an outcome measure for the assessment of treatment in randomized, blinded intervention trials of chronic heart failure: A systemic review. European Heart Journal, 26, 778793. doi:10.1093/eurheartj/ehi162

[7] Refsgaard, J. (2005) "This is a walking test, not a talking test": The six minute walking test in congestive heart failure. European Heart Journal, 26, 749-750. doi:10.1093/eurheartj/ehi207

[8] Reybrouck, T. (2003) Clinical usefulness and limitations of the 6-minute walk test in patients with cardiovascular or pulmonary disease. Chest, 123, 325-327. doi:10.1378/chest.123.2.325

[9] Beatty, A.L. (2012) Six-minute walk test as a prognostic tool in stable coronary heart disease. Archives of Internal Medicine, 172, 1096-1102. doi:10.1001/archinternmed.2012.2198

[10] Guimaraes, G.V., Carvalho, V.O. and Bocchi, E.A. (2008) Reproducibility of the self-controlled six-minute walking test in heart failure patients. Clinics, 63, 201-206. doi:10.1590/S1807-59322008000200008

[11] Griffin, B.P., Topol, E.J., Nair, D. and Ashley, K., Eds. (2009) Manual of cardiovascular medicine. 3rd Edition, Lippincott Williams and Wilkins, Philadelphia, 602.

[12] Mark, D.B., Hlatky, M.A., Harrell Jr., F.E., et al. (1987) Exercise treadmill score for predicting prognosis in coronary artery disease. Annals of Internal Medicine, 106, 793-800. doi:10.7326/0003-4819-106-6-793

[13] Sharma, K., Kohli, P. and Gulati, M. (2012) An update on exercise stress testing. Current Problems in Cardiology, 37, 194. doi:10.1016/j.cpcardiol.2011.11.004

[14] Gauri, A.J., Raxwal, V.K., Roux, L., et al. (2001) Effects of chronotropic incompetence and beta blocker use on the exercise treadmill test in men. American Heart Journal, 142, 136-141. doi:10.1067/mhj.2001.115788

[15] ATS Statement (2002) Guidelines for the six-minutes walk test. American Journal of Respiratory and Critical Care Medicine, 166, 111-117. doi:10.1164/ajrccm.166.1.at1102

[16] Strokengine.ca. Canadain stroke network. http://strokengine.ca/assess/module 6mwt indepth-en.ht $\underline{\mathrm{ml}}$

[17] Wu, G., Sanderson, B. and Bittner, V. (2003) The 6-minute walk test: How important is the learning effect. American Heart Journal, 146, 129-133. doi:10.1016/S0002-8703(03)00119-4

[18] De Almeida, F.G., Victor, E.G. and Rizzo, J.A. (2009) Hallway versus treadmill 6-minute-walk tests in patients with chronic obstructive pulmonary disease. Respir Care, 54, 1712-1716.

[19] Miyamoto, S., Nagaya, N., Satoh, T., Kyotani, S., Sakamaki, F., Fujita, M., et al. (2000) Clinical correlates and prognostic significance of six-minute walk test in patients with primary pulmonary hypertension, comparison with cardiopulmonary testing. American Journal of Respiratory and Critical Care Medicine, 161, 487-492. doi:10.1164/ajrccm.161.2.9906015

[20] Guazzi, M., Dickstein, K., Vicenzi, M. and Arena, R. (2009) Six-minute walk test and cardiopulmonary exercise testing in patients with chronic heart failure, a comparative analysis on clinical and prognostic insights. Circular Heart Failure, 2, 549-555. 